\title{
243 UNDERSTANDING AND OVERCOMING THE MECHANISM OF RESISTANCE TO ANTI-PD-1 MONOTHERAPY IN BRAIN METASTASIS
}

Laura Falceto Font*, Catherine Flores, Jack Figg, Bayli DiVita Dean, Connor Francis, Carmelle Kuizon, Ginger Moore. University of Florida, Gainesville, FL, USA

Background The average overall survival for patients with brain tumors is only 8 to 12 months. For example, the 5 -year survival rate for adults over 40 years old living with Glioblastoma multiforme, a type of brain tumor, is only $6 \%$. The brain is also a common organ for metastasis deriving from tumors such as breast cancer. The field of cancer immunotherapy has been making efforts to develop strategies that can target these brain metastases more efficiently than the standard of care in the clinic. Immune checkpoint inhibitors such as anti-PD-1 (anti-programmed cell death-1) therapy are under investigation to treat brain metastases. However, several studies have shown brain metastases to be resistant to anti-PD-1 monotherapy. Our findings have shown that a hematopoietic stem cell (HSC) transfer can overcome this resistance in brain metastasis. Our goal is to elucidate and overcome the mechanism of resistance to anti-PD-1 therapy in brain metastasis.

Methods We performed both orthotopic mammary fat pad and intracranial (IC) tumor implantations in C57/BL6J mice using a murine breast cancer cell line (E0771). Three days after tumor implantation, we administered the first of four doses of anti-PD-1 therapy delivered five days apart from each other. We measured survival and chemokine (from blood samples) differences between treatments.

Results We have observed that orthotopic mammary fat pad tumors are responsive to anti-PD-1 alone. Interestingly, when tumors derived from the same breast cancer cell line are implanted into the brain, they become non-responsive to antiPD-1 monotherapy. We have also discovered that performing a hematopoietic stem cell transfer overcomes resistance to anti-PD-1 therapy in brain metastases (figure 1). Furthermore, in our preliminary studies we have found that both CD8+ and $\mathrm{CD} 4+\mathrm{T}$ cells are required for the protective effects of anti-PD-1 against E0771 tumors.
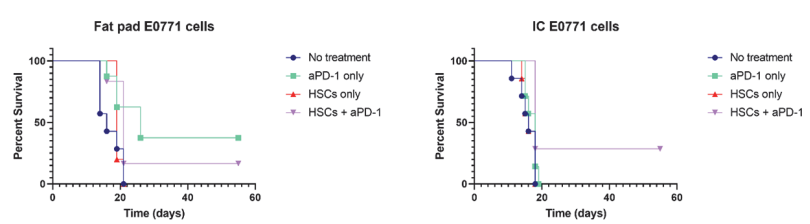

Abstract 243 Figure 1 A hematopoietic stem cell transfer overcomes resistance to anti-PD-1 in brain metastasis

Conclusions Brain metastasis are resistant to anti-PD-1 monotherapy. We have found that a hematopoietic stem cell transfer overcomes resistance to anti-PD-1 in brain metastasis. We hypothesize that, while peripheral $\mathrm{T}$ cells successfully remove breast tumors, they fail to mount anti-tumor responses in breast metastatic brain tumors. Therefore, the next step is to understand the mechanism of resistance to anti-PD-1 monotherapy in brain metastasis and to further elucidate the type of $\mathrm{T}$ cell immune responses required to overcome this resistance in brain metastasis.

Ethics Approval IACUC Protocol \#201910777 